\title{
A PRELIMINARY ESTIMATION OF THE DIRECT ULTRAVIOLET SPECTRAL IRRADIANCE IN VALENCIA (SPAIN): COMPARISON WITH MEASURED VALUES
}

\author{
F. Tena, R. Pedrós, L. Hernández, M. P. Utrillas and J. A. Martínez-Lozano \\ Departamento de Termodinámica, Universitat de València \\ 46006 Burjassot (Valencia), Spain
}

\begin{abstract}
UVSPEC and SMARTS2 models have been used to estimate the UV spectral irradiance values in Valencia, Spain, and the results of these estimations have been compared with experimental measurements of direct irradiance at normal incidence obtained using an Optronic OL 754 in the 300-400 nm range. The relative RMSD and MBD parameters have been used to estimate the deviations of the modelled values with respect to the experimental values. The results indicate that the deviations are smaller when the SMARTS2 model is used with the appropriate aerosol coefficients.
\end{abstract}

\section{INTRODUCTION}

The detection of the thinning of the ozone layer leading to the appearance of an ozone hole in the Antarctic in 1985 and the progressive ozone reduction in the following years ${ }^{(1,2)}$ stimulated the scientific community to investigate the consequences of this phenomenon, especially the increase in incident ultraviolet radiation at ground level. Over the past few years major efforts have been made to assess the trends in the increase of the UV radiation and the influence that different atmospheric parameters exert on the transmission of UV solar radiation through the atmosphere.

In order to carry out such analyses spectroradiometry can be a useful tool for studying the UV radiation characteristics at ground level, especially in relation to the biological effects of increased UV radiation which are known to be strongly wavelength dependent. Unfortunately most spectroradiometers are too expensive or have poor accuracy in the UV range and consequently experimental solar irradiance spectral data at ground level are scarce. To remedy this a number of atmospheric algorithms have been described in the literature dating back to the early 1980s which try to simulate and predict irradiance values ${ }^{(3)}$. Such algorithms can be classified into two different groups: sophisticated rigorous codes (or radiative transfer codes, RTC) and simple transmittance parameterisations (parameterised models, PM). All these models are subject to several uncertainties - usually divided into numerical errors and uncertainties in the input data which must be quantified by comparison with experimental data.

In this paper the estimated values produced by two of these models are compared with the experimental results determined from spectral irradiance measurements at Valencia, a Spanish Mediterranean coastal site. The experimental data have been obtained by means of an

Contact author E-mail: tenaf@uv.es
Optronic OL 754 spectroradiometer with a high resolution in the UV spectral range. The models employed were the UVSPEC (RTC) ${ }^{(4)}$ and the SMARTS2 (PM) ${ }^{(5)}$ which are summarised below. To evaluate the deviation of the values computed by the models from the experimental data, the relative RMSD and MBD statistical parameters have been used.

The work presented here should only be considered as a preliminary analysis of the use of the two models in the UV range, the work chiefly considers the influence of the choice of the aerosol model on their precision. It is not an exhaustive analysis of the models' validity since this would require a larger spectral data base covering a wide variety of sky conditions ${ }^{(6)}$.

\section{THE UVSPEC AND SMARTS2 MODELS}

The UVSPEC code was developed by Kylling in the University of Alaska and is available on the World Wide $\mathrm{Web}^{(4)}$. The UVSPEC code covers the wavelength region $176-850 \mathrm{~nm}$ and evaluates both direct and diffuse irradiances with $1 \mathrm{~nm}$ resolution. The radiative transfer equation is numerically integrated with DISORT $^{(7)}$, SDISORT (Pseudo-spherical or Spherical DISORT version) ${ }^{(8)}$ or TWOSTR (TWO-Stream code $)^{(9)}$. Mayer et $a l^{(6)}$ have proposed a modification of the original UVSPEC, the TWODIS, which combines the benefits of TWOSTR and DISORT. The UVSPEC uses the standard atmosphere profile USSA and the aerosol models of Shettle ${ }^{(10)}$. The UVSPEC allows the surface albedo, the cloud type, the ozone concentration, and other conditions to be varied. The different atmosphere types and the temperature, pressure and ozone profiles can also be modified if the corresponding data are available.

The SMARTS2 model (Simple Model for the Atmospheric Radiative Transfer of Sunshine) was proposed by Gueymard in $1995^{(5)}$. It is based on an extensive revision of the algorithms used to calculate direct beam radiation with the spectral model SMARTS ${ }^{(11)}$ and consists of a separate parameterisation of the different 
extinction processes ocurring in the atmosphere. To make this parameterisation, accurate transmittance functions for all atmospheric extinction processes are introduced and the effects of temperature and humidity are considered. The model also includes very accurate absorption coefficients derived from spectroscopic data. The version used in this work was kindly provided by its author and corresponds to the latest revised version (Gueymard, 1997, personal communication). SMARTS2 code allows the introduction of ground meteorological data or the choice of ten different reference atmospheres if ground data are not available. It also allows the user to choose from nine predefined aerosol models or to introduce their own aerosol coefficients. The aerosol models available in the present version are: (a) four proposed by Shettle and Fenn ${ }^{(12)}$; (b) two proposed by Braslau and Dave ${ }^{(13)}$; and (c) three corresponding to the Standard Radiation Atmosphere (SRA) ${ }^{(14)}$.

Both models allow the introduction of the spectroradiometer slit function in order to compare the estimations with experimental measurements.

\section{INSTRUMENTATION AND METHODOLOGY}

Spectral solar irradiance measurements were obtained using two commercial spectroradiometers, an Optronic 754 and a Licor Li-1800. The spectral band of the OL 754 ranges from $250 \mathrm{~nm}$ to $800 \mathrm{~nm}$, with a $2 \mathrm{~nm}$ bandwidth. The measurements can be made with minimum pass bands of $0.05 \mathrm{~nm}$. The OL 754 is a double monochromator with holographic gratings of 1200 lines. $\mathrm{mm}^{-1}$. The input optics is an integration sphere covered by PTFE and the detector is a silicon photodiode with a photomultiplier with temperature stabilisation. The spectral band of the Li-1800 ranges from $300 \mathrm{~nm}$ to $1100 \mathrm{~nm}$, with a $6 \mathrm{~nm}$ bandwidth. The characteristics of this instrument are detailed elsewhere ${ }^{(15)}$. The spectroradiometers are absolutely calibrated every three months in our laboratory by means of $200 \mathrm{~W}$ reference lamps, the Optronic with a 754 OL 752-10E (Optronic), and the Li-1800 with a $1800 /$ ORL815 (Licor).

The OL 754 has been used to obtain direct spectral irradiance measurements at normal incidence in the UV range, $280-400 \mathrm{~nm}$. The $\mathrm{Li}-1800$ has been used to determine the aerosol characteristics by means of direct spectral irradiance measurements in the visible (400$670 \mathrm{~nm}$ ). These aerosol characteristics were then introduced into the models in order better to approximate the actual atmospheric conditions. For the direct sun measurements the spectroradiometers were oriented manually by means of a three-axis ball and socket joint and alignment system. For the direct irradiance measurements, radiance limiting tubes (collimators) with a field of view of $4.7^{\circ}$ were used. Simultaneously with the spectral irradiance measurements, the total atmospheric content of ozone and water vapour were also measured with a Microtops II. Other parameters such as temperature and pressure were routinely recorded.

All the measurements corresponded to clear days and were taken on the terrace ( $40 \mathrm{~m}$ above sea level) of the Faculty of Physics, Valencia University, in the Burjassot Campus, located in the outskirts of the city of Valencia (Spain). The latitude is $39.5^{\circ} \mathrm{N}$ and the longitude is $0^{\circ}$. The obstructions above the horizon were less than $4^{\circ}$, except in a small zone in the Northwest. A previous paper describes site obstructions and the measuring setup in detail ${ }^{(16)}$.

From the spectral measurements at normal incidence in the 400-670 $\mathrm{nm}$ range obtained with the Licor, values of the total optical thickness for all the different atmospheric extinction processes, $\tau_{\mathrm{T} \lambda}$ were deduced by using the Beer law. Once the total atmospheric optical thickness had been determined, the value of the aerosol optical thickness, $\tau_{\mathrm{a} \lambda}$, was obtained by removing the contributions due to Rayleigh scattering and to absorption by the other atmospheric components from the total transmitance ${ }^{(17)}$. Applying the power law relationship proposed by Angstrom ${ }^{(18,19)}$

$$
\tau_{\mathrm{a \lambda}}=\beta \lambda^{-\alpha}
$$

$\alpha$ and $\beta$ were evaluated and used as inputs in the spectral irradiance models ${ }^{(20)}$.

In order to analyse the deviation of the values estimated by both models from the experimental values, two different statistical estimators which are commonly used for evaluating the accuracy of models have been used: RMSD (root mean square deviation) and MBD (mean bias deviation). RMSD always gives positive values whilst MBD, defined as modelled minus observed, may be positive or negative, with the positive values corresponding to overestimation by the model.

\section{RESULTS AND DISCUSSION}

Our analysis of the results was limited to establishing general comparisons between the spectral values of the experimental direct irradiance and those obtained by the models. The UVSPEC and SMARTS2 models were applied to evaluate the direct irradiance at normal incidence on four clear days in July 1999. Forty two spectral series, corresponding to solar zenith angles varying between $20^{\circ}$ and $60^{\circ}$ have been compared. For each of these measurements the $\alpha$ and $\beta$ Angstrom coefficients have been evaluated from the irradiance values in the visible range, following the methodology described above. The spectral irradiance values have been compared in the range $300-400 \mathrm{~nm}$ with a $1 \mathrm{~nm}$ pass band.

For the estimation by the SMARTS2 model, the Angstrom turbidity coefficient $\beta$ and three aerosol models, rural (SFR), urban (SFU) of Shettle and Fenn ${ }^{(12)}$ and urban (SRAU) of SRA ${ }^{(14)}$ have been used. These models were selected because they produced the best results in a previous work carried out in Valencia ${ }^{(17)}$. As an example, Figure 1 shows the experi- 
mental and the estimated values obtained by employing these three aerosol models for a solar zenith angle of $20^{\circ}$ on 15 July. The representative curves show that the model that best fits with the experimental values was the aerosol urban model. The MBD values of the three curves were $3.9 \%$ (SFU), 9.5\% (SFR) and $12.7 \%$ (SRAU). The corresponding values of the RSMD were 8.7\% (SFU), 11.8 (SFR) and $14.2 \%$ (SRAU).

The UVSPEC model takes as input the Angstrom $\alpha$ and $\beta$ coefficients and an aerosol model. The Shettle urban, rural and marine models have been used. Figure 2 presents the results obtained for the same day and for the same solar zenith angle considered for the SMARTS2. As can be observed, the curves corresponding to the values deduced by employing the different aerosol models practically coincide and the MBD $(6.3 \%)$ and the RSMD (15.5\%) were practically the same for the three models considered, varying only in the second decimal digit between one aerosol model and another. The same happened with all the spectral measurements analysed. Indeed, it appeared that, for the version used (libRadtran 0.14), the UVSPEC was almost completely insensitive to the choice of aerosol model when the $\alpha$ and $\beta$ Angstrom coefficients were used as input parameters. This observation, however, should be more carefully analysed in the future based on a higher number of experimental measurements.

For the whole set of available measurements the aver-

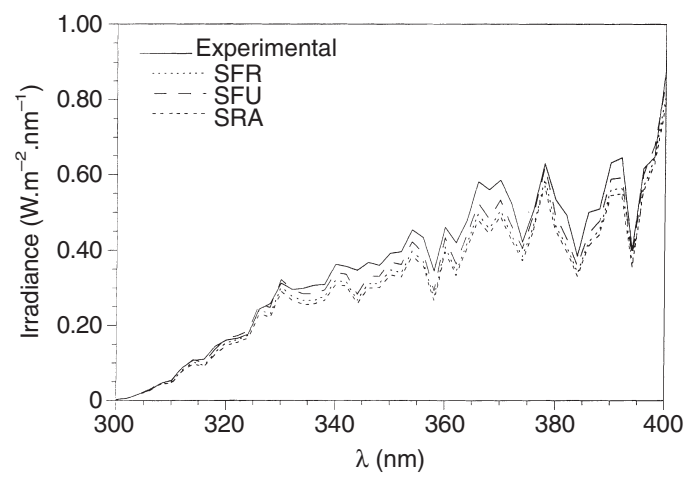

Figure 1. Comparison between the experimental data and the values estimated by the SMARTS2 code for a solar zenith angle of $20^{\circ}$ on 15 July 1999. age values of the deviations were the following: (a) for the UVSPEC the value of the MBD was $7.4 \pm 0.1 \%$, depending on the aerosol model used, and the RSMD was $15.7 \%$, independent of the aerosol model; (b) the SMARTS2 was more sensitive to the aerosol model, with values of $5.6 \%$ (MBD) and 9.4\% (RMSD) obtained for the SFU model, 11.5\% (MBD) and 13.3\% (RMSD) for the SFR model and 14.8\% (MBD) and 15.9\% (RMSD) for the SRAU model.

As was noted at the beginning of the paper, the results presented here must be interpreted with caution because they are only a first approximation to the use of the models and not an analysis of their validity. Nevertheless it is possible to point out three qualitative characteristics of the models used: (a) SMARTS2, with the appropriate aerosol model, produces estimates nearer to the experimental measurements than UVSPEC; (b) the version of the UVSPEC used, with the Angstrom coefficients as input parameters, seems to be insensitive to the choice of aerosol model; and (c) both SMARTS2 and UVSPEC systematically underestimate the direct irradiance values.

\section{ACKNOWLEDGEMENTS}

This work was supported by the Ministerio de Educación (CLI97-0345-C05) and the Generalitat Valenciana (GV97-RN-14-18).

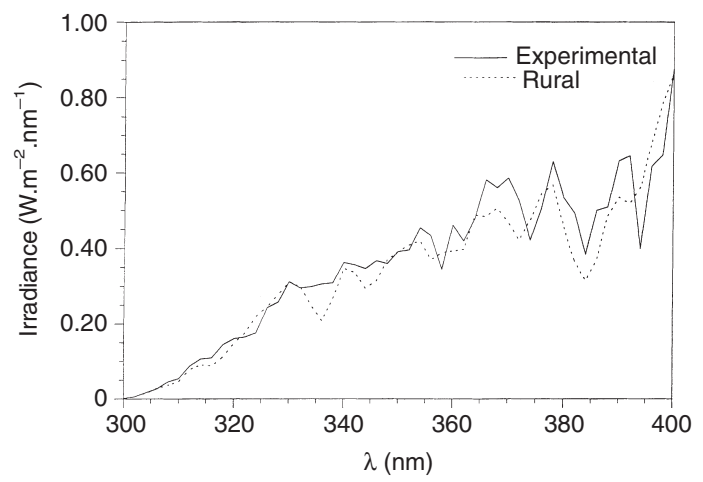

Figure 2. Comparison between the experimental data and the estimated values by the UVSPEC code for a solar zenith angle of $20^{\circ}$ on 15 July 1999.

\section{REFERENCES}

1. Bojkov, R. D. and Fioletov, V. E. Estimating the Global Ozone Characteristics during the Last 30 Years J. Geophys. Res. 100, 16537-16551 (1995).

2. Stolarsky, A. S., Bojkov, R. D., Bishop, L., Zerefos, C., Stachelin, J. and Zawodny, J. Measured Trends in Stratospheric Ozone. Science 256, 343-349 (1992).

3. Koepke, P. and 23 others. Comparison of Models used for UV Index Calculations. Photochem. Photobiol. 67, 657-662 (1998).

4. Kylling, A. UVSPEC. A Program for Calculation of Diffuse and Direct UV and Visible Fluxes and Intensities at Any Altitude. Available on $<\mathrm{ftp}$ ///kaja.gi.alaska.edu/pub/arve $>$ (1995).

5. Gueymard, C. SMARTS2, a Simple Model of the Atmospheric Radiative Transfer of Sunshine: Algorithms and Performance Assessment. FSEC-PF-270-95 (Florida Solar Energy Center, Cocoa, FL) (1995). 
F. TENA, R. PEDRÓS, L. HERNÁNDEZ, M. P. UTRILLAS and J. A. MARTÍNEZ-LOZANO

6. Mayer, B., Seckmeyer, G. and Kylling, A. Systematic Long-term Comparison of Spectral UV Measurements and UVSPEC Modeling Results. J. Geophys. Res. 102, 8755-8767 (1997).

7. Stamnes, K., Tsay, S., Wiscombe, W. and Jayaweera, K. Numerically Stable Algorithm for Discrete-ordinate-method Radiative Transfer in Multiple Scattering and Emiting Layered Media. Appl. Opt. 27, 2502-2509 (1988).

8. Dahlback, A. and Stamnes, K. A New Spherical Model for Computing the Radiation Field Available for Photolysis and Heating at Twilight. Planet. Space Sci. 39, 671-683 (1991).

9. Kylling, A., Seckmeyer, G. and Mayer, B. A Reliable and Efficient Two-stream Algorithm for Spherical Radiative Transfer: Documentation of Accuracy in Realistic Layered Media. J. Atmos. Chem. 21, 115-150 (1995).

10. Shettle, E. P. Models of Aerosol, Clouds and Precipitation for Atmospheric Propagation Studies. In: Atmospheric Propagation in the UV, Visible, IR and mm-region and Related System Aspects. AGARD Conf. Proc. 15, 1-15, 13 (1989).

11. Gueymard, C. Development and Performance Assessment of a Clear Sky Spectral Radiation Model. In: Proc. Solar '9322nd ASES Conf., Washington D.C. (1993).

12. Shettle, E. P. and Fenn, R. W. Models for the Aerosol of the Lower Atmosphere and the Effects of Humidity Variations on their Optical Properties. AFGL-TR-79-0214 Environmental Res. Paper no 676. Hanscom, AFL (Mass) (1979).

13. Braslau, N. and Dave, J.V. Effect of Aerosols on the Transfer of Solar Energy through Realistic Model Atmospheres. J. Appl. Meteorol. 30, 601-619 (1973).

14. WMO. A Preliminary Cloudless Standard Atmosphere for Radiation Computation. World Climate Programme, WCP-112. WMO/TD-No 24 (Geneva, Switzerland: World Meteorological Organization, (1986).

15. Cachorro, V. E., Utrillas, M. P., Martinez-Lozano, J. A. and De Frutos, A. A Preliminary Assessment of a Detailed Two Stream Short-wave Narrow-band Model using Spectral Radiation Measurements Solar Energy 60, $265-273$ (1997).

16. Utrillas, M.P., Martinez-Lozano, J.A. and Casanovas, A.J. Evaluation of models for estimating solar irradiation on vertical surfaces at Valencia, Spain Solar Energy 47, 223-229 (1991).

17. Utrillas, M. P., Boscá, J. V., Martínez-Lozano, J. A., Cañada, J., Tena, F. and Pinazo, J.M. A comparative study of SPECTRAL2 and SMARTS2 parametrized models based on spectral irradiance measurements at Valencia (Spain) Sol. Energ. 63, 161-171 (1998).

18. Angstrom, A. On the Atmospheric Transmission of Sun Radiation and on the Dust in the Air Geografis. Annal. 11, 156166 (1929).

19. Angstrom, A. On the Atmospheric Transmission of Sun Radiation. Geografis. Annal. 12, 130-159 (1930).

20. Martínez-Lozano, J.A., Utrillas, M.P., Tena, F. and Cachorro, V. The Parametrization of the Atmospheric Aerosol Optical Depth using the Angstrom Power Law. Sol. Energ. 63, 303-311 (1998). 